\title{
Palynological Studies to Determine Pollen Resources of Bombus haemorrhoidalis Smith
}

\author{
Narinderjit Singh, Kiran Rana*, Harish K. Sharma, Meena Thakur and R.K. Thakur \\ Department of Entomology, Dr YS Parmar University of Horticulture and Forestry, \\ Nauni, Solan-173230, Himachal Pradesh, India \\ *Corresponding author
}

\author{
A B S T R A C T
}

\begin{tabular}{|l|}
\hline Ke y w or d s \\
Bombus \\
haemorrhoidalis, \\
Pollen plants, \\
Pollen loads, \\
Pollen analysis. \\
\hline Article Info \\
\hline $\begin{array}{l}\text { Accepted: } \\
\text { 26 August } 2017 \\
\text { Available Online: } \\
\text { 10 September } 2017\end{array}$ \\
\hline
\end{tabular}

Palynological studies of pollen loads were carried out to determine the pollen sources for bumble bees under mid-hill conditions of Himachal Pradesh during 2016. The pollen loads were collected from queens and workers of $B$. haemorrhoidalis captured with the help of an insect net and then were released. A total of 73 pollen loads were collected. Microscopic preparations were made and palynological analysis was carried out. The analysis showed the occurrence of 68 pollen types served as pollen sources for bumble bee from amongst the fruit trees, medicinal plants, ornamentals, vegetable crops, weeds and wild trees. Out of these, 21 pollen types which formed homogenous pollen loads were considered as principal forage plants namely Solanum melongena, S. lycopersicum, Capsicum annuum, Digitalis purpurea, Digitalis lanata, Oenothera biennis, Martynia annua, Hibiscus syriacus, Agapanthus umbellatus, Salvia moorcroftiana, Moluccella laevis, Cassia sophera, C. fistula, Clitoria ternatea, Actinidia deliciosa, Peltophorum ferrugineum, Lupinus hartwegii, Dahlia imperialis, D. pinnata, Zinnia elegans and Justicia adhatoda.

\section{Introduction}

Bumble bees (Bombus spp.) are beneficial insects belonging to order Hymenoptera (Family: Apidae). They have important advantages in their ability to visit flowers in closed spaces (Biliński, 1973, 1976), buzz mechanism while collecting pollen from anthers (Buchmann, 1983), and especially long proboscis in some species (Sladen, 1912). These are important pollinators of crops especially where honey bees (Apis spp.) are ineffective or their activity is limited by adverse climatic conditions. They are particularly important pollen vectors for many entomophilous crops and wild flowers which require cross pollination, and can also improve success rates in partly self-fertile or wind pollinated species (Corbet et al., 1991). These are ecologically as well as economically important pollinators in cool and temperate crops and also act as model organisms in specific research (Ayasse and Jarau, 2014). Bumble bees are known for providing a service of significant pollination as their pollen loads carry a greater dry mass than those of honey bees (Broussard et al., 2011).Bumblebees require pollen for their reproduction as it is the sole protein source for developing larvae, and recent evidence suggests that adult workers have an ongoing need for pollen throughout their lives (Smeets 
and Duchateau, 2003). The composition of social bee's corbicular pollen loads contains information about both the bee's foraging behavior and the surrounding floral landscape (Marchand et al., 2015).

Palynology is the study of pollen grains produced by seed plants and spores. Pollens can be used to determine foraging resources, pollination mechanisms, migration routes and source zones of insects and pollinators (Jones and Jones, 2001). The palynological analysis of pollen loads allows the identification of plant species visited by bees for pollens and this method is more efficient than visual observation based methods because it allows the identification of a greater number of visited plant species with lower labour inputs involved (Teper, 2005). The palynological studies of pollen loads of bumble bees in different parts of the world have been carried out but no such type of work has been done in our country. Keeping all this in view, the present investigation was undertaken to determine the pollen sources of bumble bee (Bombus haemorrhoidalis) under mid-hill conditions of Himachal Pradesh.

\section{Materials and Methods}

\section{Preparation of standard pollen slides}

The standard pollen slides were prepared by using the fresh and mature flowers of Nauni area. Pollen grains from fresh flowers were placed on microscopic slide and few drops of ethyl alcohol (96\%) were dropped on slide. The fat substances appeared on the slide after dropping/pouring alcohol, were cleaned with the help of blotting paper. Then microscopic slides were treated with 1-2 drops of acetolysis mixture. This mixture was prepared fresh at every time by mixing 9 parts of acetic anhydride with 1 part of sulfuric acid. Then the content on microscopic slides was lightly warmed on alcoholic lamp so that it could not get darker. The content was washed up with ethyl alcohol (70\%) and fixed with D.P.X mountant by placing a cover slip over it.

\section{Collection of pollen loads from corbicula of bumble bee while visiting flowers}

Foraging bumble bee queens and workers of B. haemorrhoidalis with pollen loads were captured with the help of an insect net on different sampling dates at 15 days interval from February onwards throughout the year. Pollen loads were carefully removed with a dissecting needle into an individual specimen tubes and the bees were released unharmed. The microscopic preparations of pollen loads were made on the same day or were put in well labeled vials under refrigeration for preparation of slides later on.

\section{Preparation of pollen slides}

Microscopic preparations of pollens from pollen loads were made by using acetolysis method given by Avetisjan (1950). Pollen load was placed on microscopic slide and few drops of $96 \%$ ethyl alcohol were dropped on it. The fat substances appeared on the slide after pouring alcohol was cleaned gently with blotting paper. Few drops of freshly prepared acetolysis mixture were added on to the slides which comprised of acetic anhydride mixed with concentrated sulphuric acid in the ratio 9:1. Then the content on microscopic slides was lightly warmed on alcohol lamp so that it could not get darker. The content was washed up with $70 \%$ ethyl alcohol and mounted with D.P.X. The prepared slides were studied under light microscope for morphological studies and photomicrograph of pollen grains was taken. The measurement of pollen grains was taken with "Magnus Pro" software. The pollen grains were divided into five categories on basis of size of pollen grains as per classification given by Sawyer (1981). 
Table.1 Description of pollen grains found in bumble bee pollen loads and their morphology

\begin{tabular}{|c|c|c|c|c|c|c|c|c|}
\hline $\begin{array}{l}\text { Sr. } \\
\text { No. }\end{array}$ & $\begin{array}{c}\text { Common } \\
\text { name }\end{array}$ & Scientific Name & Family & $\begin{array}{l}\text { Flowering } \\
\text { Period }\end{array}$ & $\begin{array}{c}\text { Photo- } \\
\text { micrograph of } \\
\text { pollen grain }\end{array}$ & $\begin{array}{c}\text { Pollen } \\
\text { Description }\end{array}$ & $\begin{array}{l}\text { Type of } \\
\text { Pollen } \\
\text { Loads }\end{array}$ & $\begin{array}{c}\text { Habit and } \\
\text { Nature }\end{array}$ \\
\hline 1. & Field pea & Pisum sativum L. & Fabaceae & Jan-March & & $\begin{array}{l}\text { Long, } \\
\text { medium*, } \\
\text { bilateral } \\
\text { symmetry }\end{array}$ & + & $\begin{array}{l}\text { Climber, } \\
\text { vegetable }\end{array}$ \\
\hline 2. & Basuti & Justicia adhatoda L. & Acanthaceae & Feb-April & & $\begin{array}{l}\text { Long, Large, } \\
\text { radial symmetry }\end{array}$ & ++ & $\begin{array}{l}\text { Shrub, } \\
\text { Wild plant }\end{array}$ \\
\hline 3. & Mustard & $\begin{array}{l}\text { Brassica campestris } \\
\text { L. }\end{array}$ & Brassicaceae & Feb-March & & $\begin{array}{l}\text { Round, small, } \\
\text { radial symmetry }\end{array}$ & + & $\begin{array}{l}\text { Herb, } \\
\text { oilseed }\end{array}$ \\
\hline 4. & Pot marigold & $\begin{array}{l}\text { Calendula officinalis } \\
\text { L. }\end{array}$ & Asteraceae & Feb-April & & $\begin{array}{l}\text { Round, small, } \\
\text { spinolous, } \\
\text { radial symmetry }\end{array}$ & + & $\begin{array}{l}\text { Herb, } \\
\text { ornamental }\end{array}$ \\
\hline 5. & Blue thistle & Echium vulgare L. & Boraginaceae & Feb-April & & $\begin{array}{l}\text { Oval, very } \\
\text { small, bilateral } \\
\text { symmetry }\end{array}$ & + & $\begin{array}{l}\text { Herb, } \\
\text { ornamental }\end{array}$ \\
\hline 6. & $\begin{array}{l}\text { Annual } \\
\text { chrysanthemu } \\
\text { m }\end{array}$ & $\begin{array}{l}\text { Glebionis coronaria } \\
\text { (L.) Cass. ex Spach }\end{array}$ & Asteraceae & Feb-April & & $\begin{array}{l}\text { Round, } \\
\text { small,spinolous, } \\
\text { radial symmetry }\end{array}$ & + & $\begin{array}{l}\text { Herb, } \\
\text { ornamental }\end{array}$ \\
\hline
\end{tabular}




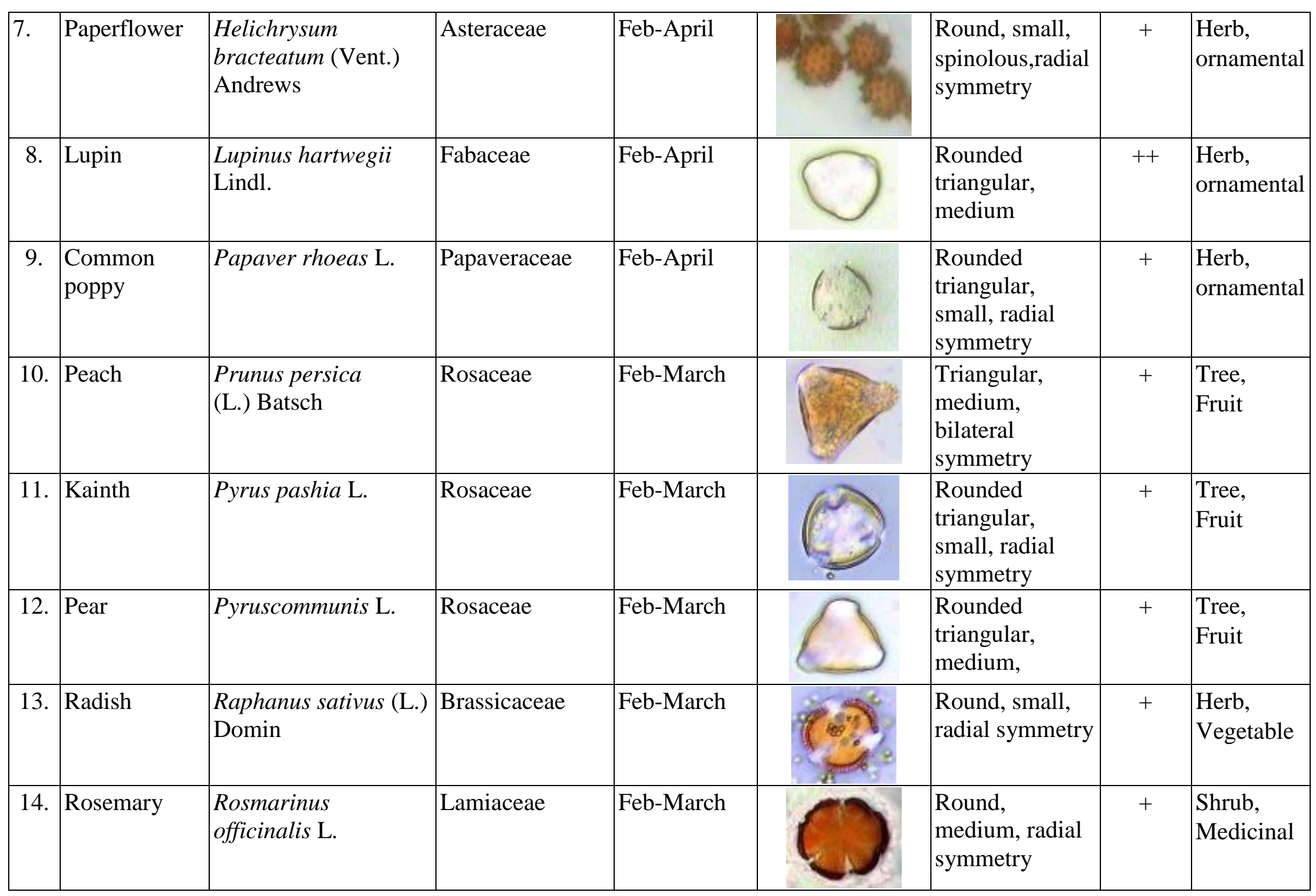




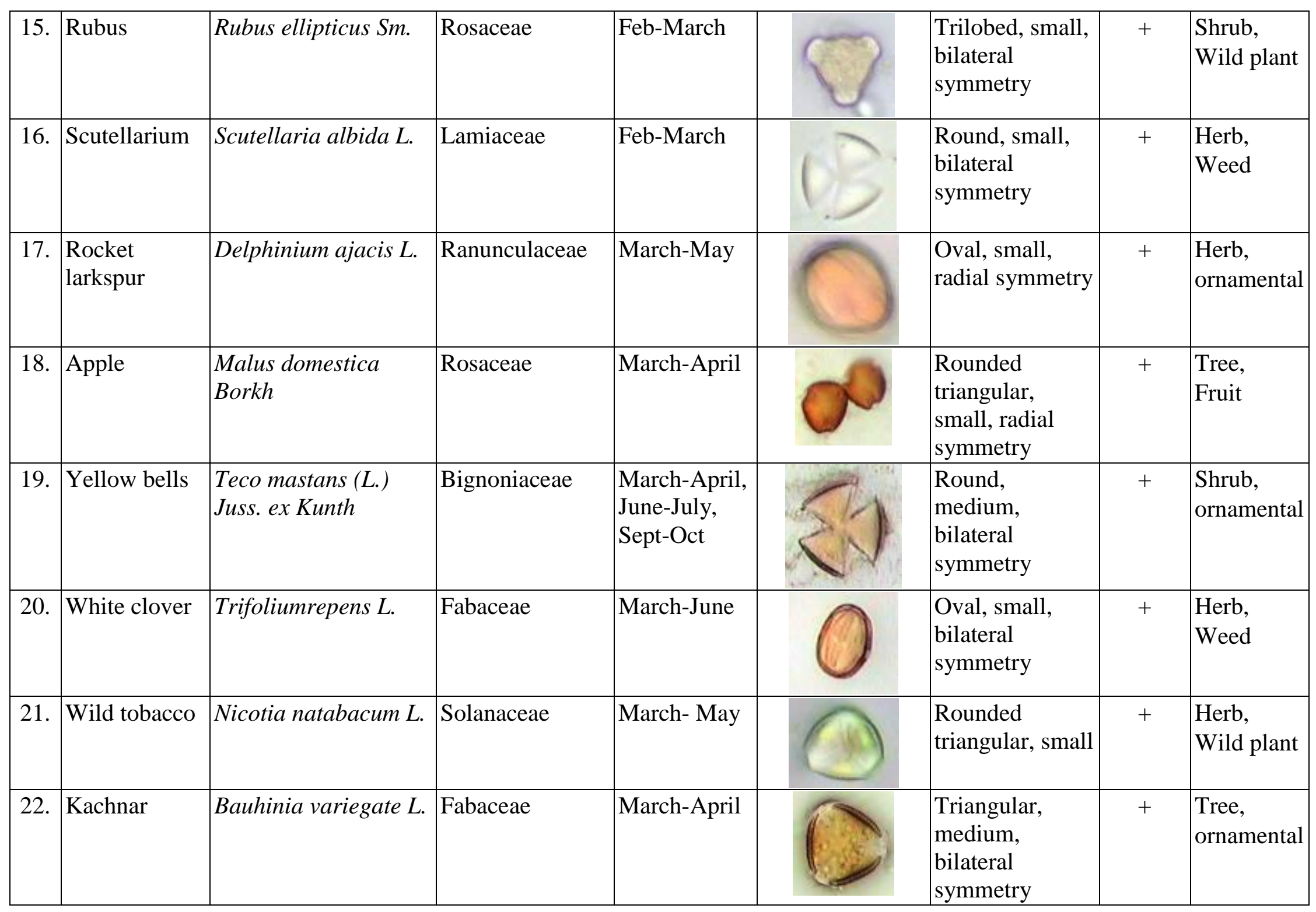




\begin{tabular}{|c|c|c|c|c|c|c|c|}
\hline 23. & $\begin{array}{l}\text { Snapdragon/ } \\
\text { Dog flower }\end{array}$ & Antirrhinum majus L. & Veronicaceae & March-May & $\begin{array}{l}\text { Rounded } \\
\text { triangular, } \\
\text { small, bilateral } \\
\text { symmetry } \\
\end{array}$ & ++ & $\begin{array}{l}\text { Herb, } \\
\text { ornamental }\end{array}$ \\
\hline 24. & Kiwifruit & $\begin{array}{l}\text { Actinidia deliciosa } \\
\text { Liang and Ferguson }\end{array}$ & Actinidiaceae & April-May & Round, small & ++ & $\begin{array}{l}\text { Climber, } \\
\text { Fruit }\end{array}$ \\
\hline 25 . & $\begin{array}{l}\text { Himalayan } \\
\text { horse chestnut }\end{array}$ & $\begin{array}{l}\text { Aesculus indica } \\
\text { (Wall. ex Camb.) } \\
\text { Hook. }\end{array}$ & Sapindaceae & April-June & $\begin{array}{l}\text { Oval, small, } \\
\text { bilateral } \\
\text { symmetry }\end{array}$ & + & $\begin{array}{l}\text { Tree, } \\
\text { ornamental }\end{array}$ \\
\hline 26. & Field thistle & Cirsium $s p$ & Asteraceae & April-June & $\begin{array}{l}\text { Round, } \\
\text { medium, } \\
\text { spinolous, }\end{array}$ & + & $\begin{array}{l}\text { Herb, } \\
\text { Weed }\end{array}$ \\
\hline 27. & $\begin{array}{l}\text { Woolly/ } \\
\text { Grecian } \\
\text { foxglove }\end{array}$ & Digitalis lanata Ehrh. & Plantaginaceae & April-July & $\begin{array}{l}\text { Round, very } \\
\text { small, }\end{array}$ & ++ & $\begin{array}{l}\text { Herb, } \\
\text { Medicinal }\end{array}$ \\
\hline 28. & $\begin{array}{l}\text { Common } \\
\text { foxglove }\end{array}$ & Digitalis purpurea L. & Plantaginaceae & April-June & Round, small & ++ & $\begin{array}{l}\text { Herb, } \\
\text { Medicinal }\end{array}$ \\
\hline 29. & Duranta & Duranta primuli $L$. & Verbenaceae & April-Oct & $\begin{array}{l}\text { Triangular, } \\
\text { small, bilateral } \\
\text { symmetry }\end{array}$ & + & \begin{tabular}{|l} 
Shrub, \\
ornamental
\end{tabular} \\
\hline 30. & Jacaranda & $\begin{array}{l}\text { Jacaranda } \\
\text { mimosifolia D.Don }\end{array}$ & Bignoniaceae & April-June & $\begin{array}{l}\text { Round, } \\
\text { medium, radial } \\
\text { symmetry }\end{array}$ & + & $\begin{array}{l}\text { Tree, } \\
\text { ornamental }\end{array}$ \\
\hline 31. & $\begin{array}{l}\text { Bells of } \\
\text { Ireland }\end{array}$ & Moluccella laevis L. & Lamiaceae & April-June & $\begin{array}{l}\text { Round, small, } \\
\text { radial symmetry }\end{array}$ & ++ & $\begin{array}{l}\text { Herb, } \\
\text { ornamental }\end{array}$ \\
\hline
\end{tabular}




\begin{tabular}{|c|c|c|c|c|c|c|c|}
\hline 32. & Pomegranate & Punica granatum $L$. & Lythraceae & April-June & $\begin{array}{l}\text { Round, small, } \\
\text { radial symmetry }\end{array}$ & + & \begin{tabular}{|l} 
Tree, \\
Fruit
\end{tabular} \\
\hline 33. & Sage & $\begin{array}{l}\text { Salvia moorcroftiana } \\
\text { Wall. exBenth. }\end{array}$ & Lamiaceae & April-May & $\begin{array}{l}\text { Round, large, } \\
\text { radial symmetry }\end{array}$ & ++ & \begin{tabular}{|l} 
Herb, \\
Medicinal
\end{tabular} \\
\hline 34. & $\begin{array}{l}\text { Kangaroo } \\
\text { apple }\end{array}$ & \begin{tabular}{|l|} 
Solanum \\
laciniatum Ait.
\end{tabular} & Solanaceae & April-Aug & \begin{tabular}{|l|} 
Oval, small, \\
radial symmetry
\end{tabular} & + & \begin{tabular}{|l|} 
Shrub, \\
Medicinal
\end{tabular} \\
\hline 35. & Chicory & Cichorium intybus L. & Asteraceae & May-June & $\begin{array}{l}\text { Trilobed,spinol } \\
\text { ous, medium, } \\
\text { bilateral } \\
\text { symmetry }\end{array}$ & + & $\begin{array}{l}\text { Herb, } \\
\text { Medicinal }\end{array}$ \\
\hline 36. & $\begin{array}{l}\text { Blue African } \\
\text { lily }\end{array}$ & $\begin{array}{l}\text { Agapanthus } \\
\text { umbellatus L. }\end{array}$ & Amaryllidaceae & May-July & $\begin{array}{l}\text { Boat shaped, } \\
\text { large, bilateral } \\
\text { symmetry }\end{array}$ & ++ & $\begin{array}{l}\text { Herb, } \\
\text { ornamental }\end{array}$ \\
\hline 37. & Capsicum & \begin{tabular}{|l} 
Capsicum \\
annuumvargrossum \\
(L.) Sendt.
\end{tabular} & Solanaceae & May-July & $\begin{array}{l}\text { Trilobed, small, } \\
\text { bilateral } \\
\text { symmetry }\end{array}$ & ++ & \begin{tabular}{|l|} 
Shrub, \\
Vegetable
\end{tabular} \\
\hline 38. & $\begin{array}{l}\text { Rose of } \\
\text { Sharon }\end{array}$ & Hibiscus syriacus L. & Malvaceae & May-Aug & $\begin{array}{l}\text { Round, very } \\
\text { large, } \\
\text { echinateradial } \\
\text { symmetry } \\
\end{array}$ & ++ & \begin{tabular}{|l|} 
Shrub, \\
ornamental
\end{tabular} \\
\hline 39. & $\begin{array}{l}\text { Garden pink- } \\
\text { sorrel }\end{array}$ & Oxalis latifoliaKunth & Oxalidaceae & May-July & $\begin{array}{l}\text { Round, small, } \\
\text { radial symmetry }\end{array}$ & + & $\begin{array}{l}\text { Herb, } \\
\text { Weed }\end{array}$ \\
\hline
\end{tabular}




\begin{tabular}{|c|c|c|c|c|c|c|c|}
\hline 40. & Tomato & $\begin{array}{l}\text { Solanum } \\
\text { lycopersicum L. }\end{array}$ & Solanaceae & May-Oct & $\begin{array}{l}\text { Round, very } \\
\text { small, radial } \\
\text { symmetry }\end{array}$ & ++ & $\begin{array}{l}\text { Herb, } \\
\text { Vegetable }\end{array}$ \\
\hline 41. & Brinjal & $\begin{array}{l}\text { Solanum melongena } \\
\text { L. }\end{array}$ & Solanaceae & May-Oct & $\begin{array}{l}\text { Oval, small, } \\
\text { bilateral } \\
\text { symmetry }\end{array}$ & ++ & $\begin{array}{l}\text { Shrub, } \\
\text { Vegetable }\end{array}$ \\
\hline 42. & $\begin{array}{l}\text { Chinese } \\
\text { trumpet vine }\end{array}$ & $\begin{array}{l}\text { Tecoma } \\
\text { grandiflora (Thunb.) } \\
\text { Loisel. }\end{array}$ & Bignoniaceae & May-June & $\begin{array}{l}\text { Round, small, } \\
\text { bilateral } \\
\text { symmetry }\end{array}$ & + & $\begin{array}{l}\text { Climber, } \\
\text { ornamental }\end{array}$ \\
\hline 43. & Green chilly & $\begin{array}{l}\text { Capsicum } \\
\text { annиuтvarannuит L. }\end{array}$ & Solanaceae & June-Sept & $\begin{array}{l}\text { Oval, small, } \\
\text { bilateral } \\
\text { symmetry }\end{array}$ & + & $\begin{array}{l}\text { Shrub, } \\
\text { Vegetable }\end{array}$ \\
\hline 44. & Cucumber & Cucumis sativus L. & Cucurbitaceae & June-Sept & $\begin{array}{l}\text { Rounded } \\
\text { triangular, large }\end{array}$ & + & $\begin{array}{l}\text { Climber, } \\
\text { Vegetable }\end{array}$ \\
\hline 45. & $\begin{array}{l}\text { Purple } \\
\text { coneflower }\end{array}$ & $\begin{array}{l}\text { Echinacea purpurea } \\
\text { (L.) Moench }\end{array}$ & Asteraceae & June-July & $\begin{array}{l}\text { Round, small, } \\
\text { spinolous,radial } \\
\text { symmetry }\end{array}$ & + & $\begin{array}{l}\text { Herb, } \\
\text { Medicinal }\end{array}$ \\
\hline 46. & Gladiolus & Gladiolus hybrida L. & Iridaceae & June-Aug & $\begin{array}{l}\text { Long, large, } \\
\text { bilateral } \\
\text { symmetry }\end{array}$ & + & $\begin{array}{l}\text { Herb, } \\
\text { ornamental }\end{array}$ \\
\hline 47. & Bitter gourd & $\begin{array}{l}\text { Momordica charantia } \\
\text { L. }\end{array}$ & Cucurbitaceae & June-Sept & $\begin{array}{l}\text { Round, large, } \\
\text { radial symmetry }\end{array}$ & + & $\begin{array}{l}\text { Climber, } \\
\text { Vegetable }\end{array}$ \\
\hline
\end{tabular}




\begin{tabular}{|c|c|c|c|c|c|c|c|}
\hline 48. & $\begin{array}{l}\text { Evening- } \\
\text { primrose }\end{array}$ & Oenothera biennis L. & Onagraceae & June-Aug & $\begin{array}{l}\text { Trilobed, large, } \\
\text { bilateral } \\
\text { symmetry }\end{array}$ & ++ & $\begin{array}{l}\text { Herb, } \\
\text { Medicinal }\end{array}$ \\
\hline 49. & $\begin{array}{l}\text { Yellow } \\
\text { gulmohar }\end{array}$ & $\begin{array}{l}\text { Peltophorum } \\
\text { ferrugineum Benth. }\end{array}$ & Fabaceae & June-Aug & $\begin{array}{l}\text { Round, } \\
\text { medium, } \\
\text { bilateral } \\
\text { symmetry }\end{array}$ & ++ & $\begin{array}{l}\text { Tree, } \\
\text { ornamental }\end{array}$ \\
\hline 50. & French beans & Phaseolus vulgaris L. & Fabaceae & June-Sept & $\begin{array}{l}\text { Triangular, } \\
\text { medium }\end{array}$ & + & $\begin{array}{l}\text { Climber, } \\
\text { Vegetable }\end{array}$ \\
\hline 51. & Clary sage & Salvia sclarea L. & Lamiaceae & June-July & $\begin{array}{l}\text { Oval, medium, } \\
\text { radial symmetry }\end{array}$ & + & $\begin{array}{l}\text { Herb, } \\
\text { Medicinal }\end{array}$ \\
\hline 52. & Krishna neel & Anagallis arvensis $L$. & Primulaceae & June-July & $\begin{array}{l}\text { Oval, small, } \\
\text { bilateral } \\
\text { symmetry }\end{array}$ & + & $\begin{array}{l}\text { Herb, } \\
\text { Weed }\end{array}$ \\
\hline 53. & Okra & $\begin{array}{l}\text { Abelmoschus } \\
\text { esculentus (L.) } \\
\text { Moench }\end{array}$ & Malvaceae & June-Sept & $\begin{array}{l}\text { Round, very } \\
\text { large, } \\
\text { echinate,radial } \\
\text { symmetry }\end{array}$ & + & $\begin{array}{l}\text { Shrub, } \\
\text { Vegetable }\end{array}$ \\
\hline 54. & Zinnia & Zinnia elegans Jacq. & Asteraceae & July-Oct & $\begin{array}{l}\text { Round, small, } \\
\text { spinolous, } \\
\text { radial symmetry }\end{array}$ & ++ & $\begin{array}{l}\text { Herb, } \\
\text { ornamental }\end{array}$ \\
\hline
\end{tabular}




\begin{tabular}{|c|c|c|c|c|c|c|c|}
\hline 55. & Aparajita & Clitoria ternatea L. & Fabaceae & July-Sept & $\begin{array}{l}\text { Triangular, } \\
\text { large, bilateral } \\
\text { symmetry }\end{array}$ & ++ & \begin{tabular}{|l} 
Climber, \\
Medicinal
\end{tabular} \\
\hline 56. & Dahlia & Dahlia pinnata Cav. & Asteraceae & Aug-Nov & \begin{tabular}{|l|} 
Round, small, \\
spinolous,radial \\
symmetry
\end{tabular} & ++ & $\begin{array}{l}\text { Herb, } \\
\text { ornamental }\end{array}$ \\
\hline 57. & White datura & $\begin{array}{l}\text { Daturas tramonium } \\
\text { L. }\end{array}$ & Solanaceae & July-Aug & $\begin{array}{l}\text { Round, } \\
\text { medium, radial } \\
\text { symmetry }\end{array}$ & + & $\begin{array}{l}\text { Shrub, } \\
\text { Medicinal }\end{array}$ \\
\hline 58. & Basant & $\begin{array}{l}\text { Hypericum } \\
\text { perforatum L. }\end{array}$ & Hypericaceae & July-Sept & $\begin{array}{l}\text { Oval, very } \\
\text { small, bilateral } \\
\text { symmetry }\end{array}$ & + & $\begin{array}{l}\text { Herb, } \\
\text { Medicinal }\end{array}$ \\
\hline 59. & Amaltas & Cassia fistula $L$. & Fabaceae & July-Aug & $\begin{array}{l}\text { Round, small, } \\
\text { radial symmetry }\end{array}$ & ++ & $\begin{array}{l}\text { Tree, } \\
\text { ornamental }\end{array}$ \\
\hline 60. & Wild salvia & $\begin{array}{l}\text { Salvia } \\
\text { coccineaBuc'hoz ex } \\
\text { Etl. }\end{array}$ & Lamiaceae & July-Sept & $\begin{array}{l}\text { Oval, large, } \\
\text { bilateral } \\
\text { symmetry }\end{array}$ & + & $\begin{array}{l}\text { Herb, } \\
\text { Wild }\end{array}$ \\
\hline 61. & Van bhindi & \begin{tabular}{|l} 
Solanum \\
khasianum C.B. \\
Clarke
\end{tabular} & Solanaceae & July-Aug & $\begin{array}{l}\text { Oval, small, } \\
\text { bilateral } \\
\text { symmetry }\end{array}$ & + & $\begin{array}{l}\text { Herb, } \\
\text { Medicinal }\end{array}$ \\
\hline 62. & Kasunda & $\begin{array}{l}\text { Cassia sophera } \\
\text { (L.)Roxb }\end{array}$ & Fabaceae & July-Sept & $\begin{array}{l}\text { Rounded } \\
\text { triangular, small }\end{array}$ & ++ & \begin{tabular}{|l} 
Shrub, \\
Wild plant
\end{tabular} \\
\hline
\end{tabular}




\begin{tabular}{|c|c|c|c|c|c|c|c|}
\hline 63. & $\begin{array}{l}\text { Roxburgh'sfol } \\
\text { dwing }\end{array}$ & $\begin{array}{l}\text { Dicliptera } \\
\text { bupleuroides L. }\end{array}$ & Acanthaceae & Aug-Nov & $\begin{array}{l}\text { Long, medium, } \\
\text { bilateral } \\
\text { symmetry }\end{array}$ & + & $\begin{array}{l}\text { Herb, } \\
\text { Weed }\end{array}$ \\
\hline 64. & $\begin{array}{l}\text { Common } \\
\text { morning- } \\
\text { glory }\end{array}$ & $\begin{array}{l}\text { Ipomoea } \\
\text { purpurea (L.) Roth }\end{array}$ & Convolvulaceae & Aug-Sept & $\begin{array}{l}\text { Round, very } \\
\text { large, radial } \\
\text { symmetry }\end{array}$ & + & $\begin{array}{l}\text { Climber, } \\
\text { Weed }\end{array}$ \\
\hline 65. & Cat's claw & Martynia annua L. & Martyniaceae & Aug-Oct & $\begin{array}{l}\text { Round, large, } \\
\text { radial symmetry }\end{array}$ & ++ & $\begin{array}{l}\text { Herb, } \\
\text { Medicinal }\end{array}$ \\
\hline 66. & $\begin{array}{l}\text { Cotton } \\
\text { rosemallow }\end{array}$ & Hibiscus mutabilis L. & Malvaceae & Sept-Oct & $\begin{array}{l}\text { Round, very } \\
\text { large,echinate } \\
\text { radial symmetry }\end{array}$ & + & \begin{tabular}{|l|} 
Tree, \\
ornamental
\end{tabular} \\
\hline 67. & Dronpushpi & $\begin{array}{l}\text { Leucas cephalotes } \\
\text { (Roth) Spreng. }\end{array}$ & Lamiaceae & Sept-Oct & $\begin{array}{l}\text { Round, small, } \\
\text { radial symmetry }\end{array}$ & + & $\begin{array}{l}\text { Herb, } \\
\text { Medicinal }\end{array}$ \\
\hline 68. & Tree dahlia & $\begin{array}{l}\text { Dahlia imperialis } \\
\text { Roezl ex Ortgies }\end{array}$ & Asteraceae & Nov-Dec & $\begin{array}{l}\text { Round, small, } \\
\text { radial symmetry }\end{array}$ & ++ & $\begin{array}{l}\text { Tree, } \\
\text { ornamental }\end{array}$ \\
\hline
\end{tabular}

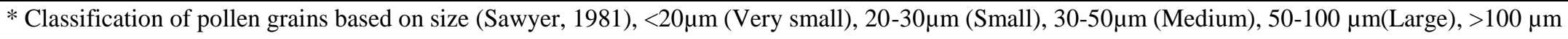
(Very large), + Multifloral pollen loads, ++ Unifloral pollen loads 


\section{Results and Discussion}

Pollen analysis of pollen loads recorded 68 plant species belonging to 27 botanical families as pollen source to bumblebees throughout their active season during 2016 under mid hill conditions of Himachal Pradesh (Table 1). The most dominant pollen types belonged to family Asteraceae (9), Fabaceae (9), Solanaceae (8) and Lamiaceae (7). These are distributed to $51 \%$ herbs, $19 \%$ shrubs, $18 \%$ tree species and $12 \%$ climbers. Twenty one pollen loads were found to be homogeneous (one-species). These plant species were considered as principle forage plants of B. haemorrhoidalis namely Solanum melongena, S. lycopersicum, Capsicum annuum, Digitalis purpurea, D. lanata, Oenothera biennis, Martynia annua, Hibiscus syriacus, Agapanthus umbellatus, Salvia moorcroftiana, Moluccella laevis, Cassia sophera, C. fistula, Clitoriaternatea, Actinidia deliciosa, Peltophorum ferrugineum, Lupinushar twegii, Dahlia imperialis, D. pinnata, Zinnia elegans and Justicia adhatoda.

The pollen morphology varies among different plant species; occur in varying shapes and sizes. They also show variation in symmetry, exine structure and sculpture. A great variation was observed in pollen types of the plant species belongs to family Fabaceae. The plant species belonging to family Asteraceae have spinolous and small pollen grains whereas in family Malvaceae pollen grain types are echinate and large in size. Pisum sativum, Justicia adhatoda Dicliptera bupleuroides and Gladiolus hybrid pollens are long and have bilateral symmetry. The pollen grains of both species of Tecoma (Bignoniaceae) are tricolporate and bilateral. Pollen grains of plants of Rosaceae family were triangular and trilobed having small to medium size. The pollen grains of plant species of Cucurbitaceaeare large, round and triangular. Pollen grains of Agapanthus umbellatus L. (Amaryllidaceae) are boat shaped and bilateral. The pollen morphology is useful to identify various species and taxa in their respective families (Shubharani et al., 2013).Pollen study have significant application in recognition of bee plants (Noor et al., 2009).

Analysis of pollen loads reveals that this region is rich in bee pollen plants. The flowering plants of an area having good value as bee pasture are necessary for development of bee colonies. Bumble bees visited these plants extensively for development and colony multiplication. It is a known fact that, due to bee activity farmers are benefitted tremendously because of the ample presence of bee foraging plants in the vicinity of their farms as bee pollination increase the crop yield in a kind of mutualistic relationships (Sahli and Conner, 2007). According to Thakur (2012) in India, about 80 percent or more of the crop plants are dependent on insect pollination. The identification and propagation of bumble bee flora will help in improving the development of bombiculture. University of Horticulture and Forestry, Nauni Solan is the pioneer research institute for developing technology for laboratory rearing of bumble bee in the country. This study will also be useful for conservation and multiplication of economically important multipurpose plants.

\section{Acknowledgement}

Authors are thankful to All India Coordinated Research Project on honey bees and pollinators for providing financial and technical help.

\section{References}

Avetisjan, B.M., 1950. Uproshennijaceto linijmetodobrabotnikipilci. 
Botanicheskii Zhurnal, 35: 385-386.

Ayasse, M., and Jarau, S. 2014.Chemical ecology of bumble bees. Annual Review of Entomology. 59: 299-319.

Biliñski, M., 1973. Praktyczna metodauzyskiwaniarodzintrzmieli do zapylaniaroœlinuprawnych. Zesz. Probl.Post.NaukRol. 131: 177-182.

Biliñski, M., 1976. Chówtrzmieli w izolatorach. Pszczeln. Zesz. Nauk. 20: 41-68.

Broussard, M., Rao, S., Stephen, W.P. and White, L. 2011.Native bees, honeybees, and pollination in Oregon Cranberries. Hortscience. 46: 885-888.

Buchmann, S.L., 1983. Buzz pollination in angiosperms. In: Jones CE, Little RJ, editors. Handbook of experimental pollination biology. New York: Van Nostrand Reinhold; pp. 73-113.

Corbet, S.A., Williams, I.H., and Osborne, J.L. 1991.Bees and the pollination of crops and wild flowers in the European Community. Bee World. 72: 47-59

Jones, G.D., and Jones, S.D. 2001. The use of pollen and its implication for Entomology. Neotropical Entomology 30: 314-349.

Marchand, P., Harmon-Threatt, A.N. and Chapela, I. 2015.Testing models of bee foraging behavior through analysis of pollen loads and floral density data. Ecological Modelling, 313: 41-49.

Noor, M.J., Khan, M.A. and Camphor, E.S. 2009.Palynological analysis of pollen loads from pollen sources of honeybees in Islamabad, Pakistan. Pakistan Journal of Botany. 41: 495-501.

Sahli, H.F., and Conner, J.K. 2007. Visitation, effectiveness and efficiency of 15 genera of visitors to wild radish, Raphanus raphanistrum (Brassicaceae). American Journal of Botany. 94: 203209.

Sawyer, R., 1981.Pollen identification for beekeepers. University College Cardiff Press, Cardiff, U.K. pp. 14-15

Shubharani, R., Roopa, P. and Sivaram, V. 2013.Pollen morphology of selected bee forage plants. Global Journal of BioScience and Biotechnology. 2: 82-90.

Sladen, F.W.L., 1912. The bumble bee life history and how to domesticate it. London: Mac Millan, 253p.

Smeets, P., and Duchateau, M.J. 2003. Longevity of Bombus terrestris workers (Hymenoptera: Apidae) in relation to pollen availability, in the absence of foraging. Apidologie. 34: 333-337.

Teper, D., 2005. Comparison of food plants of Bombus terrestris L. and Bombus lapidarius L. based on pollen analysis of their pollen loads. Journal of Apicultural Science. 49: 43-50.

Thakur, M., 2012. Bees as Pollinators Biodiversity and Conservation. International Research Journal of Agricultural Science and Soil Science. 2(1): 1-7.

\section{How to cite this article:}

Narinderjit Singh, Kiran Rana, Harish K. Sharma, Meena Thakur and Thakur, R.K. 2017. Palynological Studies to Determine Pollen Resources of Bombus haemorrhoidalis Smith. Int.J.Curr.Microbiol.App.Sci. 6(9): 2590-2602. doi: https://doi.org/10.20546/ijcmas.2017.609.319 